\title{
Stern-Gerlach Experiment
}

\author{
Maria Arazo ${ }^{1}$, Marc Barroso ${ }^{1}$, Óscar De la Torre ${ }^{1}$, Laura Moreno ${ }^{1}$, Ariadna \\ Ribes $^{1}$, Patricia Ribes ${ }^{1}$, Ana Ventura ${ }^{1}$, and David Orellana-Martín ${ }^{2}$ \\ 1 Universitat de Barcelona \\ Email: \{ maria.arazo, marc.barroso4, oscar.delatorre.perez, 95morenolaura, \\ aribesmetidieri, ribesmetidieri, a.venturabarroso\} @gmail.com \\ 2 Research Group on Natural Computing \\ Department of Computer Science and Artificial Intelligence \\ Universidad de Sevilla \\ E-mail: dorellana@us.es
}

Summary. This work is about modelling an experiment composed by multiple SternGerlach devices using Membrane Computing. We will study the behaviour of a set of independent particles passing through three linked Stern-Gerlach devices and discarting the spin down particles after passing through the first one, taking profit of the Membrane Computing's ability of running parallel processing. Using a cell-like model to describe the system and testing it using the P-lingua framework we have obtained the theorically predicted results when the number of initial multisets is high enough.

\section{Introduction}

In 1998 Gheorghe Păun introduced an alternative computing science paradigm, Membrane Computing ( $M C$ from now on) [4]. P-systems appeared, and with it, an innovative way to interpret the natural world. Those models are based on the structure of living cells and how they process compounds within their membranes (cell-like system) or even how they interact one with the others (tissue-like system). To model the processes occurring inside them, they make use of rules, which represent the different reactions or exchanges of the objects inside or through the membranes. Those rules can be of several types: communication, rewriting, annihilation, etc. For years, they have been applied to study the evolution of biological systems, neuronal systems or even complex ecosystems. Nevertheless, the application of Membrane Computing paradigms in other fields has not been yet so promoted. Therefore, one of the aims of this project is to extend the Membrane Computing applicability to physics. Moreover, this article has been used as a excuse in order to learn about this new computing paradigm and to be able to seek for possible further applications. 


\subsection{Combining $M C$ with Physics}

When studying Physics the aim is not to know exactly what things are, but to understand how they behave, this is the reason why it is so important to model natural phenomena. The modelling process' goal is to attain a set of analytical expressions that describes the studied system reduced into a determined approximation.

Because of the complexity of those analytical expressions, it is commonly useful to solve them with numerical methods. When dealing with a great number of particles, even with $n \rightarrow \infty$, being $n$ the number of particles, the computation time could become large enough that it would turn inefficient (to use certain numerical methods). Here is where Membrane Computing provides a really suitable framework, due to maximal parallelism, one of $M C$ 's main features.

Membrane Computing is originally based in a model analogous to cells and tissues. Because of that, it could fit perfectly in a system of discrete particles. By making the analogy with the structure provided by $M C$, one could identify particles with the objects, whereas rules applied in each membrane enable us to model particles' behaviour. It is also a useful tool when working with problems involving non-deterministic processes, i.e. those that can be found in modern physics, such as quantum mechanics or nuclear physics, where probabilities play an important role.

\subsection{Introduction to Quantum Mechanics}

For many years, the world was ruled by Classical Mechanics, which considered that all processes occurred in a deterministic way, i.e. one could predict the position and momentum of any particle at the same time. It was not until 1900, when Max Planck published his paper [6], that new phenomena which did not coincide with classical physics stopped seeming unwarranted, then quantum physics was borned.

Quantum Mechanics is a fundamental branch of Physics that explains the behaviour of subatomic particles and it is grounded on the idea that measurable observables are discrete and quantified. In Quantum Mechanics, the mathematical formalism is based on the Hilbert space, hence the Quantum world is described by six postulates, whereas the evolution of the body's movement in classical mechanics is ruled by Newton's Law.

Postulate 1. On the representation of the state of a physical system.

The maximum possible information on a physical system at a given time $t$ is its quantum state $\psi$, which is represented as a vector $|\psi\rangle$ of unitary module and arbitrary phase in a separable Hilbert space.

Postulate 2. On the representation of measurable magnitudes.

Every measurable magnitude of the system has associated a linear and autoadjoint operator defined on the vector space of the states. The totality of the eigenvalues is the spectrum, and the eigenvectors define a base on the Hilbert space.

Postulate 3. On the result of the measure.

The result of measuring the observable $A$ is one of its eigenvalues $a_{i}$ of the spectrum, the probability of obtaining the result $a_{i}$ is given by: 


$$
P_{\psi}^{\left(a_{i}\right)}=\left|\left\langle a_{i} \mid \psi\right\rangle\right|^{2}
$$

Postulate 4. On the collapse or reduction of the wave function.

Immediately after measuring the observable $A$ with result $a_{i}$, the new state of the system is $\left|a_{i}\right\rangle$, i.e. the corresponding eigenvector.

Postulate 5. On the temporal evolution.

Between measures, the system evolves according to the Schrödinger equation:

$$
i \hbar \frac{d}{d t}|\psi(t)\rangle=\mathcal{H}(t)|\psi(t)\rangle
$$

Where $\mathcal{H}$ is the Hamiltonian of the system.

Postulate 6. On the Pauli exclusion principle.

The position and momentum operators for fermions satisfy commutative rules that are directly related to the Pauli exclusion principle, i.e. that two fermions cannot have the same quantum numbers.

\section{Stern-Gerlach experiment}

\subsection{The basic Stern-Gerlach experiment}

The Stern-Gerlach experiment [3] is used to illustrate that particles have intrinsic properties such as the spin, the orbital momentum, etc. The total momentum of a particle is the composition of the orbital momentum and the spin, being the last one the observable that is measured by the Stern-Gerlach device. In particular, we focus the study on the third component of the spin that it is discrete and quantifiable, and can only take the values (i.e. eigenvalues) $+\frac{\hbar}{2}$ (denoted as up to simplify notation) or $-\frac{\hbar}{2}$ (down).

The basic Stern-Gerlach experiment is composed by a magnet that creates a non-uniform magnetic field oriented towards a general direction $\hat{n}$ that is contained in the plain surface perpendicular to the particle's direction of propagation (the $y$ axis in Figure 1). Once the particle has passed through the magnetic field, the third component of the spin may have changed.

In the particular case of a Stern-Gerlach (device) oriented towards the $z$ axis (i.e. the magnetic field too), the third component of the spin is measured after the particles passes through. While in a more general case (where $\hat{n}$ is a general direction as mentioned above), the measured magnitude is the projection of the spin in that arbitrary direction.

\subsection{The experiment modelled with $M C$}

The modelled experiment consists on three Stern-Gerlach devices situated along the $x$ axis, and a set of particles that go through the three of them and impact 
on a screen. Initially, the incident particles have an undetermined state, i.e. the third component of the spin may be positive or negative and unknown unless it is explicitly measured. The first Stern-Gerlach, which is $\hat{z}$-oriented, defines the third component of the spin with a fifty percent of probability of being either positive (or up, to simplify notation), or negative (down). Then, once a particle with non-determined spin goes through the first Stern-Gerlach device, which in Figure 2 is labelled as $S G 1$, the spin-state of the particle becomes determined. This is what we define as the initial state for the other two Stern-Gerlach devices. Since we are considering an arbitrary number $n$ of incident particles, after this step we would obtain $50 \%$ particles with third component of the spin up and $50 \%$ down if $n$ is large enough.

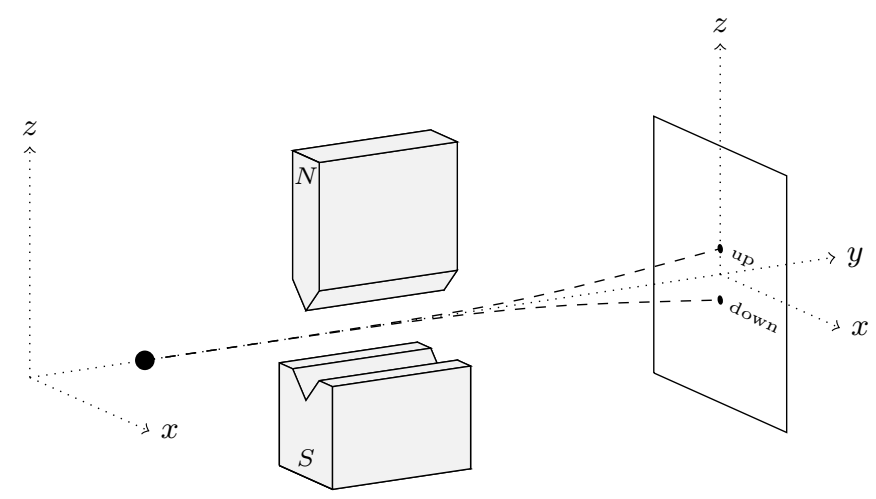

Fig. 1: Original Stern-Gerlach experiment, with the magnet parallel to the $\hat{z}$ axis so the $z$ component of the spin is measured.

For the second Stern-Gerlach device, we discard the particles with initial spin down and make the up-particles go through the magnet (labelled as $S G 2$ ), oriented with an arbitrary angle $\theta$ as shown in Figure 2. In this general case, the probabilities of obtaining spin up or spin down do not only depend on the initial state of the particle (now restrained to up), but also on the angle $\theta$ between the Stern-Gerlach device and the $z$ axis. Those probabilities are derived on the following lines.

For $\hat{n}=\sin \theta \hat{\imath}+\cos \theta \hat{k}$ the direction of the Stern-Gerlach device and $\boldsymbol{\sigma}=$ $\sigma_{x} \hat{\imath}+\sigma_{y} \hat{\jmath}+\sigma_{z} \hat{k}$ a general vector for the Pauli matrices ${ }^{3}$, the associated matrix is

${ }^{3}$ The Pauli matrices are the most general hermitic matrices of dimension $2 \times 2$ with eigenvalues 1 and -1 , and are defined as:

$$
\sigma_{x}=\left(\begin{array}{cc}
0 & 1 \\
1 & 0
\end{array}\right) \quad \sigma_{y}=\left(\begin{array}{cc}
0 & -i \\
i & 0
\end{array}\right) \quad \sigma_{z}=\left(\begin{array}{cc}
1 & 0 \\
0 & -1
\end{array}\right)
$$

With eigenvalues 1 and -1 and the corresponding eigenvectors: 


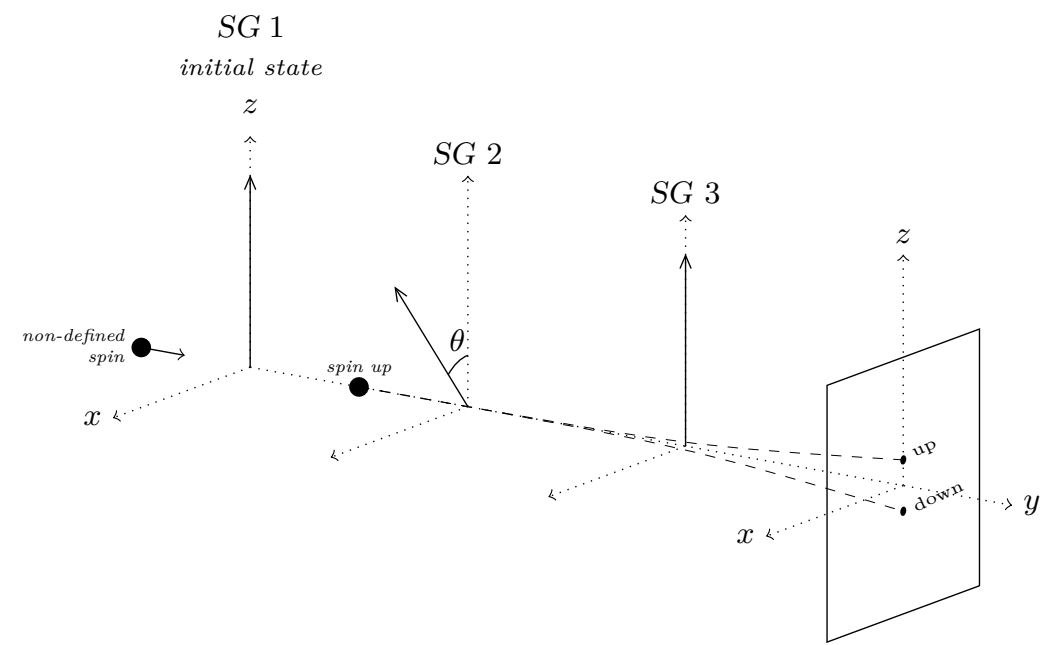

Fig. 2: Three Stern-Gerlach devices as proposed in this article

$$
\hat{n} \boldsymbol{\sigma}=\left(\begin{array}{cc}
\cos \theta & \sin \theta \\
\sin \theta & -\cos \theta
\end{array}\right)
$$

Given that the eigenvalues are +1 and -1 as in the Pauli matrices, and imposing that the corresponding eigenvectors generalised as $(\alpha \beta)$ should be normal and therefore satisfy that $|\alpha|^{2}+|\beta|^{2}=1$, the eigenvectors found are:

$$
\begin{gathered}
|\hat{n} \boldsymbol{\sigma}=+1\rangle=\left(\begin{array}{c}
\cos \theta / 2 \\
\sin \theta / 2
\end{array}\right) \\
|\hat{n} \boldsymbol{\sigma}=-1\rangle=\left(\begin{array}{c}
-\sin \theta / 2 \\
\cos \theta / 2
\end{array}\right)
\end{gathered}
$$

And the probabilities ${ }^{4}$ of obtaining spin up or down for a particle with initial up state $^{5}$ are defined by:

$$
\begin{array}{cc}
\left|\sigma_{x}=+1\right\rangle=\frac{1}{\sqrt{2}}\left(\begin{array}{l}
1 \\
1
\end{array}\right) & \left|\sigma_{x}=-1\right\rangle=\frac{1}{\sqrt{2}}\left(\begin{array}{c}
1 \\
-1
\end{array}\right) \\
\left|\sigma_{y}=+1\right\rangle=\frac{1}{\sqrt{2}}\left(\begin{array}{l}
1 \\
i
\end{array}\right) & \left|\sigma_{y}=-1\right\rangle=\frac{1}{\sqrt{2}}\left(\begin{array}{c}
1 \\
-i
\end{array}\right) \\
\left|\sigma_{z}=+1\right\rangle=\left(\begin{array}{l}
1 \\
0
\end{array}\right) & \left|\sigma_{z}=-1\right\rangle=\left(\begin{array}{c}
0 \\
1
\end{array}\right)
\end{array}
$$

${ }^{4}$ More on notation: the first subscript refers to the initial state, and the second one to the final state, so $\mathcal{P}_{\uparrow \downarrow}$ is the probability of obtaining down spin given a particle with initial spin up.

${ }^{5}$ Notation for the initial states up or down: 


$$
\begin{gathered}
\mathcal{P}_{\uparrow \uparrow}=|\langle\hat{n} \boldsymbol{\sigma}=+1 \mid+\rangle|^{2}=\left|(\cos \theta / 2 \sin \theta / 2)\left(\begin{array}{l}
1 \\
0
\end{array}\right)\right|^{2}=\cos ^{2} \frac{\theta}{2} \\
\mathcal{P}_{\uparrow \downarrow}=|\langle\hat{n} \boldsymbol{\sigma}=-1 \mid+\rangle|^{2}=\left|(-\sin \theta / 2 \cos \theta / 2)\left(\begin{array}{l}
1 \\
0
\end{array}\right)\right|^{2}=\sin ^{2} \frac{\theta}{2}
\end{gathered}
$$

Following that same procedure, the probabilities for initial down state particles are:

$$
\begin{gathered}
\mathcal{P}_{\downarrow \uparrow}=|\langle\hat{n} \boldsymbol{\sigma}=+1 \mid-\rangle|^{2}=\left|(\cos \theta / 2 \sin \theta / 2)\left(\begin{array}{l}
0 \\
1
\end{array}\right)\right|^{2}=\sin ^{2} \frac{\theta}{2} \\
\mathcal{P}_{\downarrow \downarrow}=|\langle\hat{n} \boldsymbol{\sigma}=-1 \mid-\rangle|^{2}=\left|(-\sin \theta / 2 \cos \theta / 2)\left(\begin{array}{l}
0 \\
1
\end{array}\right)\right|^{2}=\cos ^{2} \frac{\theta}{2}
\end{gathered}
$$

Finally, the particles will pass through the last SG device (SG3), which is oriented on the $z$ axis and therefore allows us to measure the third component of the $\operatorname{spin}^{6}$ and count how many particles have as final state spin up or spin down. This result, though redundant, shows one of the most important facts of Quantum Mechanics: that measure alters the system. As we can observe here, though we considered only the up-particles to go through the second Stern-Gerlach, on the final state (i.e. the screen) we have recovered the initial distribution of fifty percent of particles up and fifty percent down. See Figure 3 for an scheme of the proposed experiment.

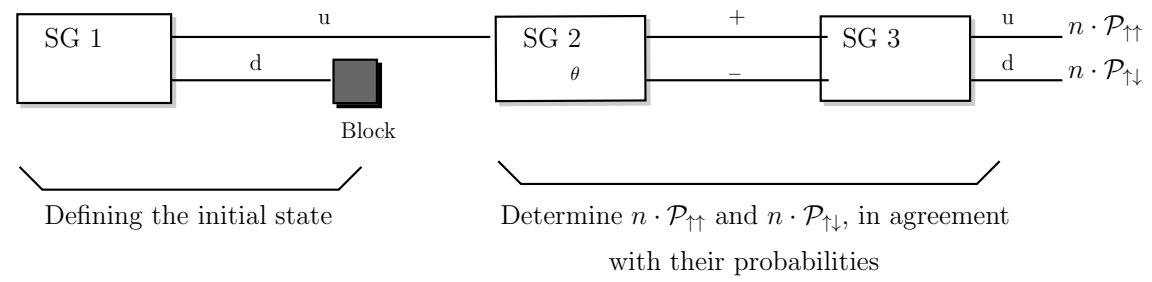

Fig. 3: Schematic description of the proposed experiment

\section{P-system model}

\subsection{PDP systems}

P systems [2] [5] are an abstraction of the membrane structure inside a cell, which delimite regions containing objects that can evolve acording to certain rules. In

$$
\text { up: } \quad|\uparrow\rangle=|+\rangle=\left(\begin{array}{l}
1 \\
0
\end{array}\right) \quad \text { down: } \quad|\downarrow\rangle=|-\rangle=\left(\begin{array}{l}
0 \\
1
\end{array}\right)
$$

${ }^{6}$ As a reminder, the Stern-Gerlach device basically measures the projection of the spin in the direction that it is oriented, i.e. a $\hat{z}$-SG measures the third component $S_{z}$ and so on. 
general, the dynamic of those systems is defined through a non-deterministic and synchronised mode. Population Dyanmics P systems (PDP) models are a complex variant of $\mathrm{P}$ systems, as they consider a collection of environments, each containing a cell (all of them with the same membrane structure and rules) connected among them as a network. Also, in those models, rules are associated with probabilistic functions and membranes with polarizations. All these ingredients make PDP systems a useful computational tool to model complex systems.

On this articles, we focus on a reduced version of PDP systems as no envrionment and polarizations are needed. What follows are the main aspects.

We define a probabilistic P system as a tuple

$$
\Pi=\left(\Gamma, \mu, \mathcal{M}_{1}, \ldots, \mathcal{M}_{q}, R,\left\{f_{r} \mid r \in R\right\}\right)
$$

where:

- $\Gamma$ is a finite set, not empty, called alphabet, whose elements are named objects of $\Pi$. The whole of all finite multisets over $\Gamma$ is denoted by $M F(\Gamma)$.

- $\mu$ is a tree structure, labelled by $\{i \mid 1 \leq i \leq q\}$, that describes the membranes' structure. The skin membrane (also named as tree root) is the only one labelled by 1 .

- $\mathcal{M}_{i} \in M F(\Gamma), 1 \leq i \leq q$, is the initial multiset of objects associated to cell $i$.

- $R$ is a finite set of evolution rules of the form: $u[v]_{i} \rightarrow u^{\prime}\left[v^{\prime}\right]_{i}$, where $u, v, u^{\prime}, v^{\prime} \in$ $M F(\Gamma), 1 \leq i \leq q$, and $|u|+|v| \neq 0$. With $u[v]_{i}$ being the left-hand side of the rule.

- For each $r \in R, f_{r} \in[0,1]$, describes the probability distribution over the rules with a same left-hand side. Then $\sum_{r} f_{r}=1$ for all the the rules in $R$ whose left-hand side is equal.

A rule $r \in R$ of the form $u[v]_{i} \rightarrow u^{\prime}\left[v^{\prime}\right]_{i}$ can be applied within a membrane labelled $i$ if it contains $v$ and its parent membrane contains $u$. If a rule of this kind is applied, objects in $v$ and $u$ vanish from membrane $i$ and its parent. Simultaneously, objects in $v^{\prime}$ and $u^{\prime}$ are included in membrane $i$ and its parent, respectively.

A configuration for any unit time is a tuple that specifies the multisets of objects that can be found in each membrane. In every step of time, rules applied are chosen in a non-deterministic way depending on its left-hand side, taking into account the probability associated to each of them. A maximal number of rules are applied simultaneously. Computation is a succession of configurations such that the first one coincides with the initial configuration and every of the remaining are obtained from the former using the rules of the system as it has been described above.

\subsection{Model}

In order to test the designed system we have define it for the simulator given by the P-lingua framework, using a single cell with two inner membranes. Given $n$ as the number of particles we want to do the experiment with, we put an object $a$ (in 
membrane 2) $n$ times, i.e., $a^{n}$ would be the input multiset.

Let $\Pi_{\mathrm{SG}}=\left(\Gamma, \mu, \mathcal{M}_{1}, \mathcal{M}_{2}, \mathcal{M}_{3}, R,\left\{f_{r} \mid r \in R\right\}\right)$, where:

- $\Gamma=\{a, u, d\}$ is the alphabet we use for this multiset.

- $\mu=\left[[]_{2}[]_{3}\right]_{1}$ is the structure of the membranes.

- $\mathcal{M}_{i}=\emptyset, i \in\{1,3\}$.

- $\mathcal{M}_{2}=a^{n}$.

- The set of rules $R$, each rule with its corresponding probability, is:

(a) These rules take care of the initial state spin of the particles that is determined by the first Stern Gerlach device. The particles with spin down are blocked.

$$
\begin{aligned}
& r_{1} \equiv[a]_{2} \stackrel{1 / 2}{\longrightarrow}[d]_{2} \\
& r_{2} \equiv[a]_{2} \stackrel{1 / 2}{\longrightarrow} u[]_{2}
\end{aligned}
$$

(b) Here, we simulate the particles passing through the second and third Stern Gerlach devices. The spin of the particles is determined according to a probability given by the angle of the magnetic field of the second Stern Gerlach with the $\hat{z}$ axis. The third Stern Gerlach is simulated by introducing the particles with definite spin within the membrane with label 3, where the results are collected.

$$
\begin{aligned}
& r_{3} \equiv u[]_{3} \stackrel{\cos ^{2}(\theta / 2)}{\longrightarrow}[u]_{3}, \\
& r_{4} \equiv u[]_{3} \stackrel{1-\cos ^{2}(\theta / 2)}{\longrightarrow}[d]_{3},
\end{aligned}
$$

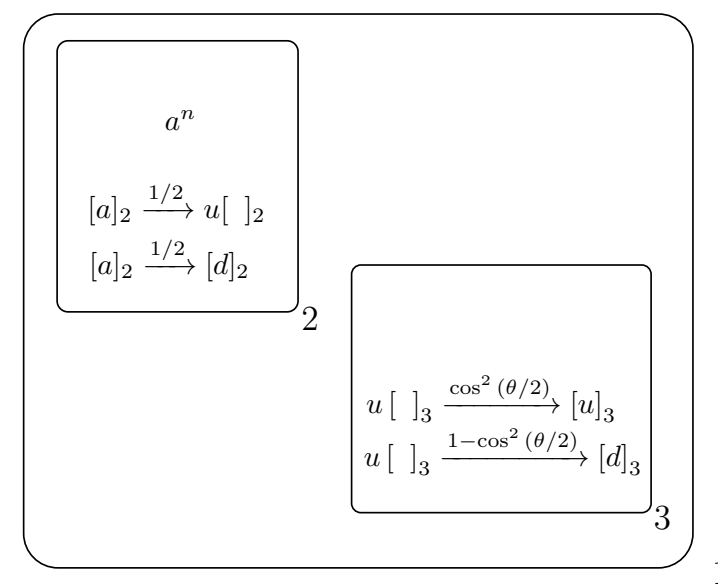

Fig. 4: Visual representation of our P-system 


\section{Code}

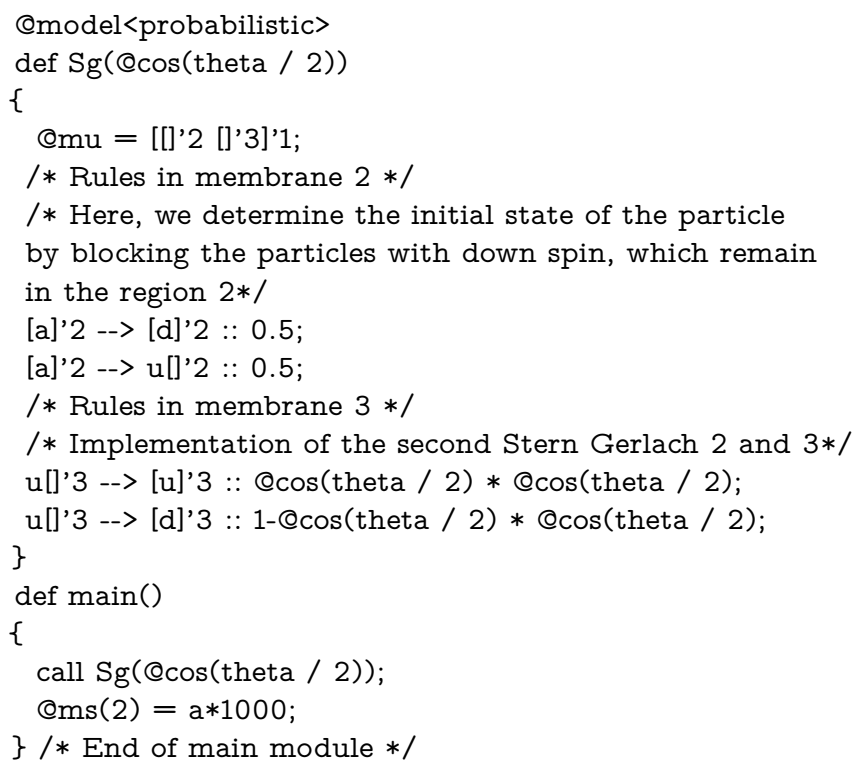




\section{Results}

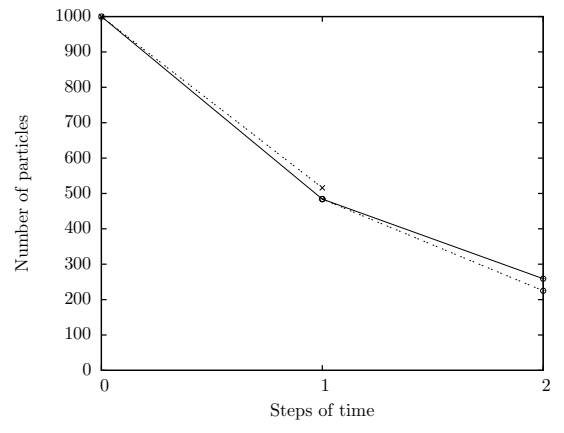

(a) $\theta=\pi / 2$.

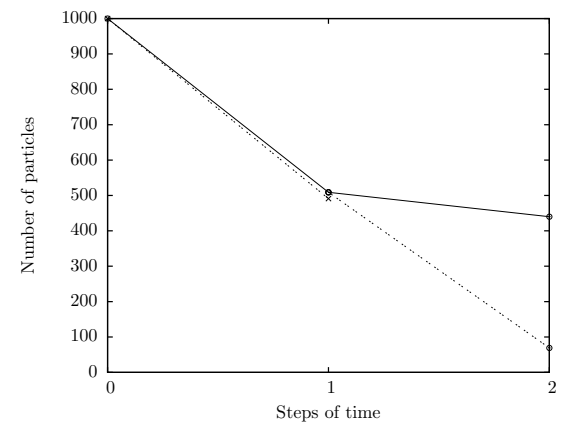

(b) $\theta=\pi / 4$.

Fig. 5: Results obtained with $n=10^{3}$ particles, for two different angles, $\theta=\pi / 2$ and $\theta=\pi / 4$. In both figures, it has been represented how from the inicial $n$ particles we obtain a first selection of up and down particles, and from the up ones how we reach the final results. In dashed lines are represented the path to down particles, whereas in solid lines to the up ones. The cross at the end of the dashed line represents the block stopping the down particles in the end of the 1st step of time, as it is shown in Figure 3.

The results obtained:

\begin{tabular}{|c|c|c|c|c|c|c|c|c|c|}
\hline & \multicolumn{8}{|c|}{ Number of initial objects, $n$} \\
\hline & & \multicolumn{2}{|c|}{$10^{1}$} & \multicolumn{2}{|c|}{$10^{2}$} & \multicolumn{2}{|c|}{$10^{3}$} & \multicolumn{2}{|c|}{$10^{4}$} \\
\hline & & $\mathrm{u}$ & $\mathrm{d}$ & $\mathbf{u}$ & $\mathbf{d}$ & $\mathbf{u}$ & d & $\mathbf{u}$ & d \\
\hline \multirow{4}{*}{$\theta$} & 0 & 4 & 0 & 49 & 0 & 489 & 0 & 4974 & 0 \\
\hline & $\pi / 4$ & 5 & 0 & 42 & 1 & 440 & 69 & 4239 & 759 \\
\hline & $\pi / 2$ & 4 & 2 & 32 & 25 & 259 & 225 & 2456 & 2586 \\
\hline & $3 \pi / 4$ & 0 & 3 & 11 & 39 & 70 & 439 & 782 & 4309 \\
\hline
\end{tabular}

Table 1: Simulated results for 4 given initial numbers of particles and for different values of the angle $\theta$.

For $\boldsymbol{\theta}=\mathbf{0}$ : As it can be seen from Table 1, the simulated results validate the expected behavior of the particles. The first Stern-Gerlach device SG1 determinates the initial 
state (spin up or down) and blocks the particles with spin down. As the two consecutive Stern Gerlach are aligned, the probability of obtaining particles with spin down in SG2 and SG3 is null. As $n \rightarrow \infty$, the probability of obtaining up spin particles tends to $\frac{1}{2}$.

For $\boldsymbol{\theta}=\frac{\pi}{4}$ : The first Stern Gerlach continues acting as a selector of the particles with spin $u p$, however the SG2 and SG3 are not aligned, allowing that approximately $7 \%$ of the particles have spin down, as it can be seen in Figure $5 \mathrm{~b}$.

For $\boldsymbol{\theta}=\frac{\pi}{2}$ : due to the block imposed by the SG1, approximately only half the particles reach SG2 and SG3, and the relative orientation of both devices causes the final proportion of both spin up and spin down particles to be approximately a quarter of the total, as it can be seen in Figure 5a.

For $\boldsymbol{\theta}=\frac{\mathbf{3} \boldsymbol{\pi}}{\mathbf{4}}$ : As expected, the numbers of resulting particles with spin $u p$ and with spin down is quite similar to the numbers obtained for $\theta=\frac{\pi}{4}$ but exchanging the results for up and down particles.

Summarizating, as the number of particles in the experiment increases, the experimental probability (calculated $P_{\uparrow \uparrow}^{\exp }=u / N$ and $P_{\uparrow \downarrow}^{\exp }=d / N$ ) tends to the expected probabilities $\left(P_{\uparrow \uparrow}=1 / 2 \cos ^{2}(\theta / 2)\right.$ and $\left.P_{\downarrow \downarrow}=1 / 2 \sin ^{2}(\theta / 2)\right)$, as shown in Table 2 .

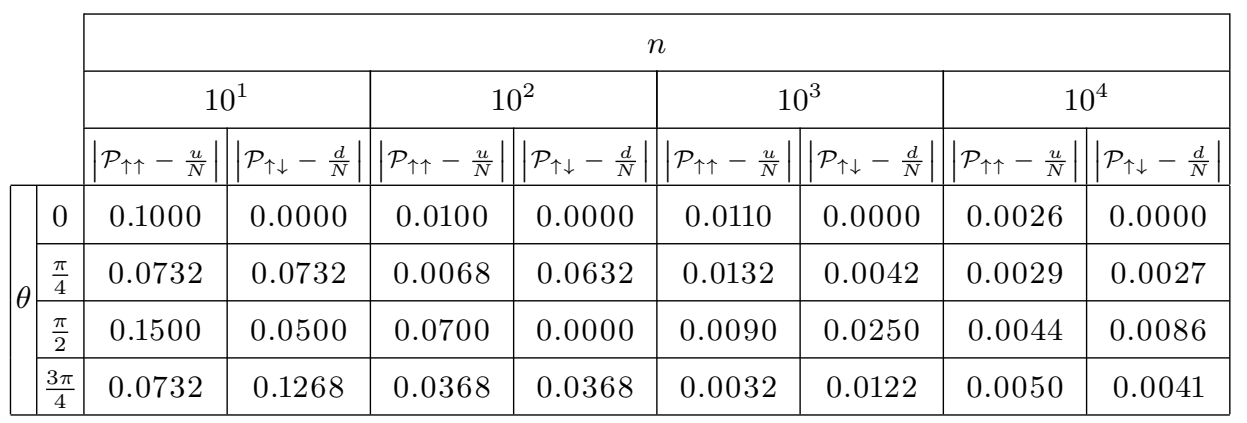

Table 2: Simulated results for 4 given initial numbers of particles and for different values of the angle $\theta$.

\section{Conclusions}

The results yielded by the designed system (obtained by the P-lingua simulator) are consistent with the theory, as have been explained above. Therefore, we have achieved our main objective: showing that $\mathrm{P}$ systems can be applied to physics, and more specifically, they can be used to implement a simplified/theoretical version of the S-G experiment, and only a little part of the power of such computational systems was used. No other exceptional consequence was predicted, as we understand that this works basically as a pedagogical application. Further research could consist in trying to apply these systems to non-trivial physical phenomena, where an analytic result might not be possible to obtain. Taking into account the non deterministic approach inherent to the model, as explained 
before, and the ability to make all the computations and apply all the rules in a parallel sequence (following the maxpar criterion), it seems a very suitable framework to implement other experiments from the modern physics world. For example, light polarization works in a similar way to the Stern-Gerlach experiment; with some modifications to the model we could simulate how light behaves when passing through a polarizer. Other applications, however, can be arbitrary hard, as the very nature of the objects used in the membranes make it very difficult to exemplify a portion of matter, for instance. Also, it is important to remark that a lot more theory about computation is developed around $M C$ that the one shown here, taking the subject as far as showing that these cell-like scheme is a universal Turing machine [1], and thus able to make any computation our normal computers can. There is no theoretical limit on what can be implemented.

\section{Acknowledgments.}

We would like to thank: Agustín Riscos-Núñez, Carmen Graciani and Mario J. PérezJiménez from Universidad de Sevilla and Ricardo Graciani from Universitat de Barcelona for their valuable comments and suggestions, as well as for their reviews of the several versions of this paper; the 14th BWMC attendants for their patience and advice on our first ideas; and also Francesc Salvat, Assumpta Parreño, Ricardo Graciani and Bruno Juliá-Díaz from Universitat de Barcelona for offering us the opportunity to attend the Brainstorming.

\section{References}

1. G. Ciobanu, G. Paun, and M. J. Pérez-Jiménez. Applications of membrane computing, volume 17. Springer, 2006.

2. M. Colomer, A. Margalida, and M. J. Pérez-Jiménez. Population Dynamics P System (PDP) Models: A Standardized Protocol for Describing and Applying Novel Bio-Inspired Computing Tools. PLOS ONE, 8(4):1-13, 2013.

3. W. Gerlach and O. Stern. Der experimentelle Nachweis der Richtungsquantelung im Magnetfeld. Zeitschrift fuer Physik, 9:349-352, 1922.

4. G. Păun. Computing with membranes. Journal of Computer and System Sciences, 61(1):108-143, 2000.

5. M. J. Pérez-Jiménez. Simulación y Análisis Computacional en Biología de Sistemas. Tema IV: Modelización computacional basada en sistemas P. Modelos estocásticos y probabilísticos.

6. M. Planck. Ueber das gesetz der energieverteilung im normalspectrum. Annalen der Physik, 309(3):553-563, 1901. 